\title{
Anaerobic growth of halophilic archaeobacteria by reduction of fumarate
}

\author{
AHARON OREN \\ The Division of Microbial and Molecular Ecology, The Institute of Life Sciences, The Hebrew University of Jerusalem, \\ Jerusalem 91904, Israel
}

(Received 26 September 1990; revised 29 November 1990; accepted 9 January 199I)

\begin{abstract}
A number of strains of halophilic archaeobacteria of the genera Halobacterium and Haloferax were able to grow anaerobically using fumarate as electron acceptor. The species showing the best anaerobic growth with fumarate were Haloferax volcanii and Haloferax denitrificans. The two Haloarcula species tested did not show anaerobic growth enhancement with fumarate. During anaerobic growth of Haloferax volcanii in the presence of fumarate, succinate accumulated in the medium with a stoichiometry of only $0.16-0.23 \mathrm{mmol}$ succinate per mmol fumarate consumed; this can be explained by the use of succinate for assimilatory purposes. The ability to reduce fumarate to succinate did not correlate with the ability to grow anaerobically using nitrate, dimethylsulphoxide or trimethylamine $\mathbf{N}$-oxide as terminal electron acceptors. Anaerobic respiration with fumarate as electron acceptor supplies the halophilic archaeobacteria with an additional mode of energy generation in the absence of molecular oxygen.
\end{abstract}

\section{Introduction}

The extremely halophilic bacteria (order Halobacteriales) are the group best adapted to aerobic life among the archaeobacteria. They possess a respiratory chain, enabling them to grow chemo-organotrophically with molecular oxygen as terminal electron acceptor.

Oxygen is often in short supply in hypersaline environments, as a result of both the frequently high microbial densities present in such habitats and the limited solubility of oxygen at high salt concentrations. Therefore, it is not surprising that representatives of the Halobacteriales have developed various modes of energy generation under anaerobic conditions, including denitrification (Mancinelli \& Hochstein, 1986), fermentation of arginine (Hartmann et al., 1980), and use of the lightdriven proton pump, bacteriorhodopsin.

We have recently shown that dimethylsulphoxide (DMSO) and trimethylamine $N$-oxide (TMAO) can serve as electron acceptors in many members of the Halobacteriaceae (Oren \& Trüper, 1990). These substrates allow anaerobic growth, which is coupled to their reduction to dimethylsulphide and trimethylamine, respectively.

In this study the ability of halophilic archaeobacteria to grow anaerobically using fumarate as the terminal

Abbreviation: TMAO, trimethylamine $N$-oxide. electron acceptor in respiration has been investigated. Anaerobic respiration using fumarate is widespread among the eubacteria (obligate as well as facultative anaerobes), and may even be used as a mechanism of short-term survival in the absence of oxygen in certain invertebrate animals (Hochachka \& Mustafa, 1972). However, its possible use as an electron acceptor by representatives of the archaeobacterial kingdom has never been reported.

\section{Methods}

Bacterial strains and aerobic culture conditions. Halobacterium halobium R1, Halobacterium halobium NRC-1 and Halobacterium salinarium 5 were grown in medium containing $\left(\mathrm{g}^{-1}\right): \mathrm{NaCl}, 250 ; \mathrm{KCl}, 5 \cdot 0$; $\mathrm{MgCl}_{2} .6 \mathrm{H}_{2} \mathrm{O}, 5 \cdot 0 ; \mathrm{NH}_{4} \mathrm{Cl}, 5 \cdot 0 ;$ yeast extract, $10 \cdot 0 ; \mathrm{pH} 7 \cdot 0$. The growth medium for Haloferax volcanii A TCC 29605 and Haloferax denitrificans ATCC 35960 contained $\left(\mathrm{g} \mathrm{l}^{-1}\right): \mathrm{NaCl}, 125 ; \mathrm{MgCl}_{2} .6 \mathrm{H}_{2} \mathrm{O}, 50 ; \mathrm{K}_{2} \mathrm{SO}_{4}$, $5 \cdot 0 ; \mathrm{CaCl}_{2} .2 \mathrm{H}_{2} \mathrm{O}, 0 \cdot 13$; yeast extract, $5 \cdot 0 ;$ Bacto-tryptone, $5 \cdot 0 ; \mathrm{pH} 7 \cdot 0$. For Haloferax mediterranei ATCC 35300 and Haloferax gibbonsii ATCC 33593 the medium contained $\left(\mathrm{g} \mathrm{l}^{-1}\right) \mathrm{NaCl}, 175 ; \mathrm{MgCl}_{2} .6 \mathrm{H}_{2} \mathrm{O}$, $20 ; \mathrm{K}_{2} \mathrm{SO}_{4}, 5 \cdot 0 ; \mathrm{CaCl}_{2} .2 \mathrm{H}_{2} \mathrm{O}, 0 \cdot 13$; yeast extract, $5 \cdot 0 ; \mathrm{pH} 7 \cdot 0$. The growth medium for Haloarcula marismortui ATCC 43049 and Haloarcula vallismortis ATCC 29715 contained $\left(\mathrm{gl}^{-1}\right): \mathrm{NaCl}, 206$; $\mathrm{MgSO}_{4} .7 \mathrm{H}_{2} \mathrm{O}, 36 ; \mathrm{KCl}, 0.37 ; \mathrm{CaCl}_{2} .2 \mathrm{H}_{2} \mathrm{O}, 0.5 ; \mathrm{MnCl}_{2}, 0.013$; yeast extract, $5.0 ; \mathrm{pH} 7 \cdot 0$. All strains were grown with shaking at $35^{\circ} \mathrm{C}$ in $250 \mathrm{ml}$ Erlenmeyer flasks containing $100 \mathrm{ml}$ of the respective growth medium.

Growth experiments in the presence of fumarate. Tubes $(25 \mathrm{ml})$ were filled to the top with the appropriate growth medium supplemented with 25 mM-PIPES buffer (Sigma), and a filter-sterilized $\mathrm{NaOH}$ - 
neutralized solution of $0.86 \mathrm{M}$-fumaric acid was added to a final concentration of $40 \mathrm{mM}$ (or otherwise as indicated), and inoculated to give an $\mathrm{OD}_{600}$ of approximately $0 \cdot 02-0 \cdot 03$. The tubes were closed with rubber stoppers, sealed with aluminium crimp seals, and incubated in the dark at $37^{\circ} \mathrm{C}$. No special attempts were made to remove any molecular oxygen that may have been present in the medium because aerobic respiration by the cells generated anaerobic conditions in the tubes: using the Winkler titration method no free oxygen could be detected in inoculated tubes after $1 \mathrm{~d}$ of incubation. The $\mathrm{OD}_{600}$ of the cultures was measured daily by inserting the tubes in a Milton-Roy Spectronic spectrophotometer (model 501) provided with a special holder to contain the tubes; $\mathrm{OD}_{600}$ readings were then converted to the corresponding values obtained in cuvettes of $1 \mathrm{~cm}$ optical pathlength. For the determination of cell protein, samples of the cultures $(2-10 \mathrm{ml})$ were filtered on glass fibre filters (Whatman GF/C), washed with $10 \mathrm{ml}$ of a salt solution identical to the growth medium without organic nutrients, and the cellular protein retained on the filters was determined by the Lowry method using bovine serum albumin as standard

In a number of experiments cells were inoculated in $75 \mathrm{ml}$ medium supplemented with 18-48 mM fumarate, under nitrogen gas in $120 \mathrm{ml}$ serum bottles sealed with rubber stoppers and aluminium crimp seals as above. Samples were withdrawn daily by means of a syringe for determination of $\mathrm{OD}_{600}$, and of fumarate and succinate concentrations.

Quantitative assays for the reduction of fumarate to succinate. The concentration of fumarate in culture supernatants was measured colorimetrically according to the Furth and Hermann reaction (Hartford, 1962). To $0 \cdot 2 \mathrm{ml}$ portions of culture supernatant in test tubes, $0.8 \mathrm{ml}$ water was added, followed by $1.3 \mathrm{ml}$ pyridine and $5.7 \mathrm{ml}$ acetic anhydride. After mixing, the tubes were incubated at $32^{\circ} \mathrm{C}$ for $30 \mathrm{~min}$, and the $\mathrm{A}_{385}$ was measured. A calibration curve was made for fumarate in the presence of $0.2 \mathrm{ml}$ uninoculated culture medium per tube, as the presence of the medium was found to interfere with the colour reaction.

Succinate was assayed colorimetrically, using the reaction of succinic anhydride with hydroquinone to form dihydronaphthazarin (Swann, 1957). The procedure was modified to yield a micro-scale determination : $0.5 \mathrm{ml}$ portions of culture supernatant were dried in test tubes in an oven at $90^{\circ} \mathrm{C} ; 0.5 \mathrm{~g}$ hydroquinone was added, followed by $2.5 \mathrm{ml}$ concentrated sulphuric acid, and the contents of the tubes were mixed. The tubes were incubated for $30 \mathrm{~min}$ at $110^{\circ} \mathrm{C}$ and for a further $2 \mathrm{~h}$ at $132^{\circ} \mathrm{C}$, during which period the contents of the tubes were mixed twice. After cooling, $3 \mathrm{ml}$ water was added, the contents of the tubes were transferred to $50 \mathrm{ml}$ test tubes, $2 \mathrm{ml}$ benzene was added to each tube, and the mixture was shaken for $30 \mathrm{~s}$. The benzene extract was separated from the aqueous phase by centrifugation, and its absorbance was measured at $520 \mathrm{~nm}$. A calibration curve was made for succinate in the presence of $0.5 \mathrm{ml}$ uninoculated culture medium in each tube.

\section{Results and Discussion}

Four of the nine strains of archaeobacterial halophiles tested grew under anaerobic conditions when the growth medium was supplemented with fumarate (Table 1). Growth in the presence of fumarate was indicated by an increase in both culture $\mathrm{OD}_{600}$ and cellular protein. In the absence of fumarate no significant growth was observed, unless alternative electron acceptors were supplied that could be used by the particular strain tested, such as DMSO, TMAO or nitrate (Oren \&

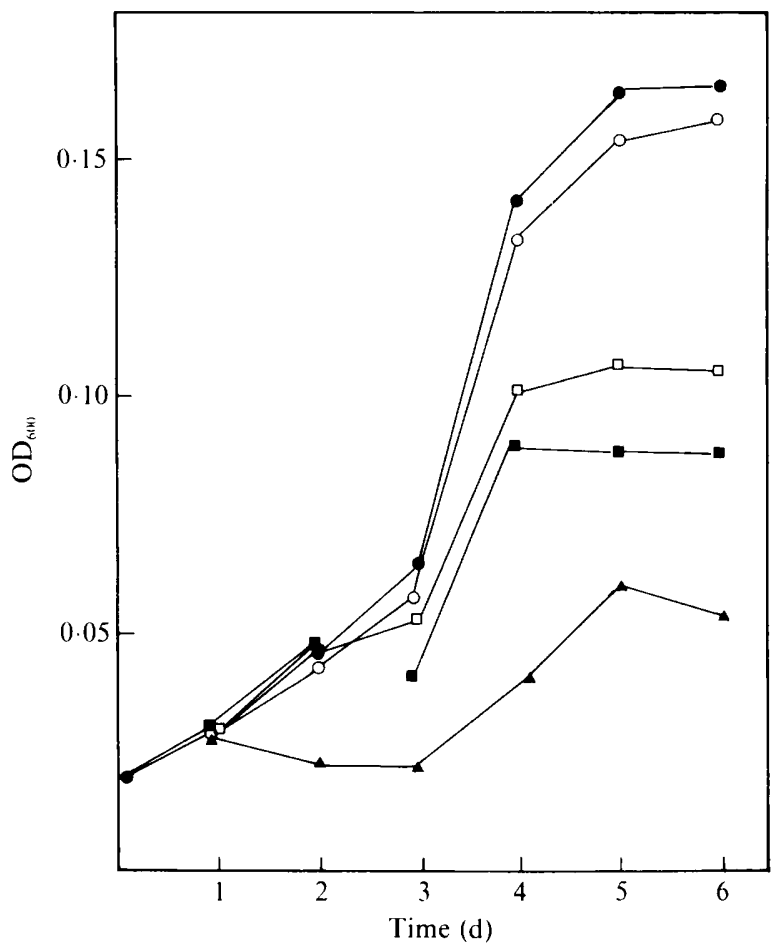

Fig. 1. Growth of Haloferax volcanii under anaerobic conditions as a function of the added concentration of fumarate in the growth medium. Cells were grown in $25 \mathrm{ml}$ tubes at $37^{\circ} \mathrm{C}$ without fumarate $(\boldsymbol{\Delta})$, or with the addition of $3.4 \mathrm{mM}^{-}(\boldsymbol{\square}), 8.6 \mathrm{mM}^{-}(\square), 17.2 \mathrm{mM}^{-}(\bigcirc)$, or $34 \mathrm{~mm}$ fumarate (O)

Table 1. Ability of different species of halophilic archaeobacteria to grow anaerobically with fumarate as the electron acceptor

An increase in cell yield of at least $100 \%$ in the presence of $40 \mathrm{~mm}$ fumarate, as compared to the control without addition of fumarate, was considered a positive growth enhancement.

\begin{tabular}{lcccc}
\hline \hline & \multicolumn{4}{c}{ Anaerobic growth with* } \\
\cline { 2 - 5 } Strain & Fumarate & DMSO & TMAO Nitrate \\
\hline Halobacterium halobium R & + & + & + & - \\
Halobacterium halobium NRC-1 & + & + & + & - \\
Halobacterium salinarium 5 & - & + & - & - \\
Haloferax volcanii & + & + & - & - \\
Haloferax denitrificans & + & + & - & + \\
Haloferax mediterranei & - & + & + & ND \\
Haloferax gibbonsii & - & - & - & - \\
Haloarcula marismortui & - & + & + & + \\
Haloarcula vallismortis & - & + & \pm & ND \\
\hline \hline
\end{tabular}

ND, Not determined.

* Data on the reduction of DMSO, TMAO and nitrate were derived from Oren \& Trüper (1990) and my unpublished results.

Trüper, 1990). When fumarate was replaced by an equivalent concentration of succinate no growth occurred (data not shown).

The ability to use fumarate as electron acceptor was 

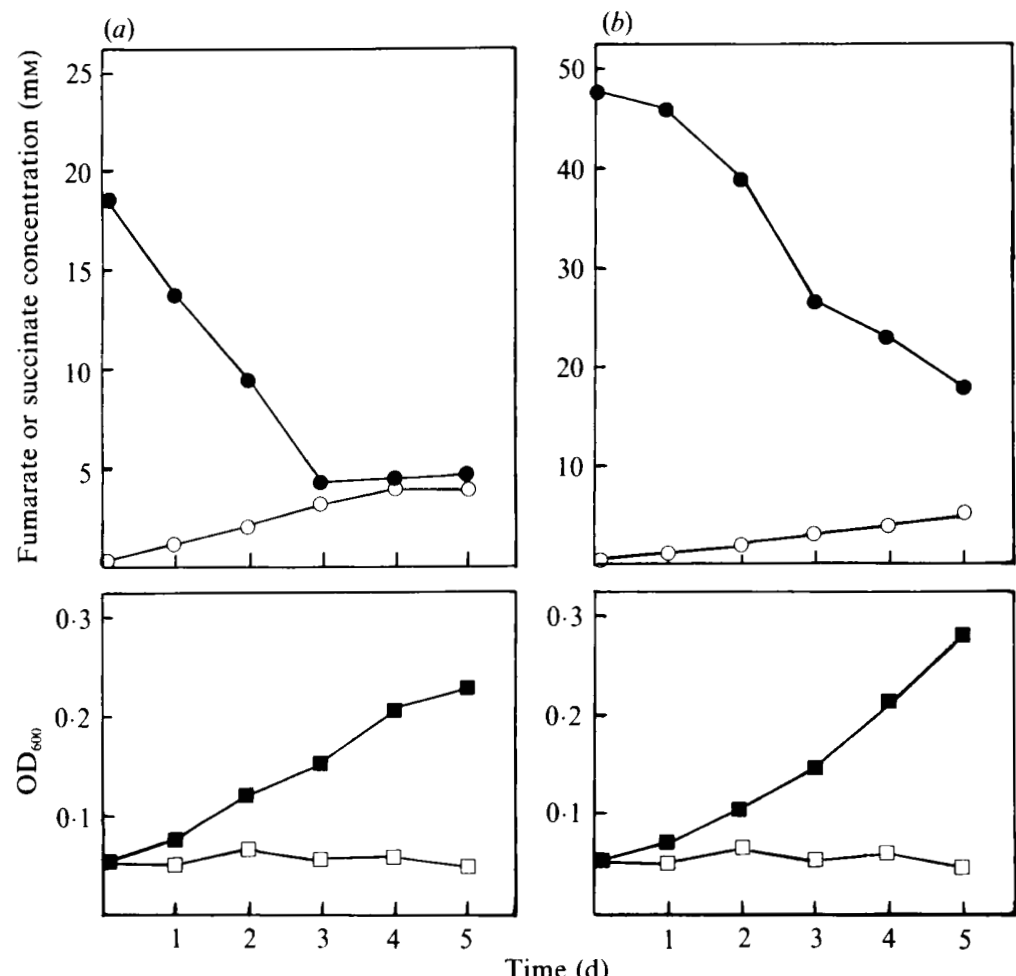

Fig. 2. Decrease in fumarate concentration and increase in succinate concentration in the growth medium during a typical anaerobic growth experiment of Haloferax volcanii. Cells were inoculated in $75 \mathrm{ml}$ medium supplemented with $18 \mathrm{mM}-(a)$ or $48 \mathrm{~mm}$-fumarate $(b)$, under nitrogen gas in $120 \mathrm{ml}$ serum bottles. Samples were withdrawn daily, and $\mathrm{OD}_{600}(\boldsymbol{\square})$, and the concentrations of fumarate $(\boldsymbol{O})$ and succinate $(O)$ in the culture supernatants were determined. The lower panels also show the $\mathrm{OD}_{600}$ of a similar culture in the absence of added fumarate $(\square)$. detected in representatives of two of the three presently recognized genera of the non-coccoid neutrophilic halophilic archaeobacteria: Halobacterium and Haloferax. Neither of the Haloarcula species tested ( $H$. marismortui and $H$. vallismortis) grew anaerobically by reduction of fumarate. The ability to grow anaerobically in the presence of fumarate did not correlate with the ability to grow anaerobically using nitrate, DMSO or TMAO as terminal electron acceptors.

In a quantitative study with Haloferax volcanii, the cell yield obtained increased with increasing fumarate concentration up to about $17 \mathrm{~mm}$ (Fig. 1). An increase of between 0.54 and $0.84 \mathrm{mg}$ cell protein per mmol of fumarate added and consumed was measured in the different experiments (compare with values of $4.3 \mathrm{mg}$ cell protein per mmol of DMSO and $2 \cdot 1 \mathrm{mg}$ cell protein per mmol TMAO for Haloferax mediterranei; Oren \& Trüper, 1990). The final growth yields obtained were somewhat variable (compare Figs 1 and 2). To examine what factors might limit anaerobic growth of Haloferax volcanii when excess fumarate $(40 \mathrm{mM})$ was added, the effect of different potential electron donors for respiration - acetate, glycerol, and L-glutamate - was examined (Kauri et al., 1990). No increase in growth yield was observed, and when the stationary growth phase was reached, between 11 and $23 \mathrm{~mm}$-fumarate remained in the culture supernatant. Changes in $\mathrm{pH}$ were probably not responsible for the termination of growth, as in none of the systems in which the buffered medium was used did the $\mathrm{pH}$ drop to values below 6.0 .

During anaerobic growth of Haloferax volcanii in the presence of fumarate, the fumarate concentration decreased, with a concomitant appearance of succinate in the culture supernatant (Fig. 2). In uninoculated control experiments no decrease in fumarate concentration was observed (data not shown). When fumarate concentrations of $20 \mathrm{~mm}$ or less were used, the onset of the stationary growth phase coincided with the depletion of fumarate in the medium; at higher fumarate concentrations other - unknown - factors limited growth (compare Figs 1 and 2). The conversion of fumarate to succinate was not stoichiometric, and only $0 \cdot 16-0.23 \mathrm{mmol}$ of succinate accumulated per mmol of fumarate consumed. The low ratio cannot be explained solely by the use of succinate as electron donor during respiration, because for every seven molecules of fumarate reduced to succinate only one molecule of succinate can be respired. This discrepancy may be accounted for by the assimilatory use of succinate or fumarate by the cells. Haloferax volcanii, as well as a variety of other halophilic archaeobacteria, grows well using succinate as the sole or supplementary carbon source (Grant \& Larsen, 1989; Kauri et al., 1990), and membrane transport systems for both succinate and fumarate have been detected in Halobacterium, Haloarcula and Halococcus strains (Plakunov et al., 1984; Zvyagintseva et al., 1984). 
Respiratory electron transport with fumarate as terminal electron acceptor in eubacteria has, in most cases, been shown to involve menaquinone (Thauer $e t$ al., 1977; Wissenbach et al., 1990). Menaquinone has a redox potential $\left(E_{0}^{\prime}=-74 \mathrm{mV}\right)$ more negative than the succinate/fumarate couple $\left(E_{0}^{\prime}=+33 \mathrm{mV}\right)$; ubiquinone has a more positive one $\left(E_{0}^{\prime}=+113 \mathrm{mV}\right)$. The reduction of fumarate with ubiquinone is therefore thermodynamically unfavourable (Thauer et al., 1977). Menaquinone is present constitutively in archaeobacteria of the Halobacterium group: halobacteria have menaquinones [MK-8 and $\left.\mathrm{MK}-8\left(\mathrm{H}_{2}\right)\right]$ as the sole respiratory quinones (Collins et al., 1981; Grant \& Larsen, 1989).

In eubacteria the fumarate reductase reducing fumarate to succinate is located at the inner side of the cytoplasmic membrane (Kröger, 1977). Thus, dissimilatory reduction of fumarate can be expected to require transport of fumarate through the cytoplasmic membrane to the site of its reduction. The presence of a transport system for fumarate has been demonstrated in strains of Halobacterium and Halococcus (Zvyagintseva et al., 1984).

It was shown in this study that, in addition to nitrate, DMSO and TMAO, several species of the Halobacteriaceae can use fumarate as an electron acceptor for respiration in the absence of molecular oxygen. This diversity of electron acceptors may provide members of this archaeobacterial group with additional modes of anaerobic survival and growth.

I thank L. I. Hochstein (Moffett Field, CA, USA) for the gift of a culture of Haloferax denitrificans, and Gilat Simon for technical assistance.

This work was supported by a grant from the Hebrew University of Jerusalem Mutual Fund.

\section{References}

Collins, M. D., Ross, H. N. M., Tindall, B. J. \& Grant, W. D. (1981). Distribution of isoprenoid quinones in halophilic bacteria. Journal of Applied Bacteriology 50, 559-565.

Grant, W. D. \& LARSEN, H. (1989). Group III. Extremely halophilic archaeobacteria. Order Halobacteriales ord. nov. In Bergey's Manual of Systematic Bacteriology, vol. 3, pp. 2216-2233. Edited by J. T. Staley, M. P. Bryant, N. Pfennig \& J. G. Holt. Baltimore: Williams $\&$ Wilkins.

HARTFORD, C. G. (1962). Rapid spectrophotometric method for the determination of itaconic, citric, aconitic, and fumaric acids. Analytical Chemistry 34, 426-428.

HartmanN, R., Sickinger, H.-D. \& Oesterhelt, D. (1980). Anaerobic growth of halobacteria. Proceedings of the National Academy of Sciences of the United States of America 77, 3821-3825.

HochachKa, P. W. \& MUSTAFA, T. (1972). Invertebrate facultative anaerobiosis. Science 178, 1056-1060.

Kauri, T., Wallace, R. \& Kushner, D. J. (1990). Nutrition of the halophilic archaebacterium, Haloferax volcanii. Systematic and Applied Microbiology 13, 14-18.

KRÖGER, A. (1977). Phosphorylative electron transport with fumarate and nitrate as terminal hydrogen acceptors. Symposia of the Society for General Microbiology 27, 61-93.

Mancinelli, R. L. \& Hochstein, L. I. (1986). The occurrence of denitrification in extremely halophilic bacteria. FEMS Microbiology Letters 35, 55-58.

OREN, A. \& TRÜPER, H. G. (1990). Anaerobic growth of halophilic archaeobacteria by reduction of dimethylsulfoxide and trimethylamine $N$-oxide. FEMS Microbiology Letters 70, 33-36.

Plakunov, V. K., Zvyagintseva, I. S. \& Tarasov, A. L. (1984). Transport of ${ }^{14} \mathrm{C}$-dicarboxylic acids by bacteria from the family Halobacteriaceae. Mikrobiologiya 53, 364-370.

SWANN, M. H. (1957). Microcolorimetric method for $o$-phthalates. Analytical Chemistry 29, 1352-1353.

Thauer, R. K., Jungermann, K. \& Decker, K. (1977). Energy conservation in chemotrophic anaerobic bacteria. Bacteriological Reviews 41, 100-180.

Wissenbach, U., Kröger, A. \& Unden, G. (1990). The specific functions of menaquinone and dimethylmenaquinone in anaerobic respiration with fumarate, dimethylsulfoxide, trimethylamine- $N$ oxide and nitrate by Escherichia coli. Archives of Microbiology 154, 60-66.

Zvyagintseva, I. S., Tarasov, A. L. \& Plakunov, V. K. (1984). Comparative characteristics of the transport systems for C4dicarboxylic acids in Halobacterium and Halococcus cultures. Mikrobiologiya 53, 520-524. 